\title{
Male breast cancer: quite rare, quite advanced, quite challenging
}

\author{
Iulian Radu ${ }^{1,2}$, Andrian Panuța ${ }^{*}$, , Mihaela Buna-Arvinte ${ }^{1}$, Cristina Terinte $^{3}$, Dan \\ Ferariu $^{3}$, Dragos Viorel Scripcariu ${ }^{1}$, Viorel Scripcariu ${ }^{1,2}$
}

${ }_{1}^{1} 1^{\text {st }}$ Surgical Oncology Unit, lasi Regional Cancer Institute, Romania, ${ }^{2}$ Department of Surgery, "Grigore T. Popa" University of Medicine and Pharmacy lasi, Romania, ${ }^{3}$ Department of Pathology, lasi Regional Cancer Institute, Romania

\begin{abstract}
Male breast cancer $(\mathrm{MBC})$ is a rare disease with peculiarities that differentiate it from female breast cancer. We present the experience of our surgical oncology unit in the management of MBC. Over a period of 4 years and 6 months 10 cases of MBC were diagnosed by physical examination, imaging (ultrasound and/or mammography) and tumor biopsy (core biopsy, fine needle aspiration or extemporaneous histologic exam), underwent surgery (mainly modified Madden radical mastectomy) performed by a single surgical team and were managed by a single oncology committee. The results of our retrospective study are similar with data revealed in literature. Two of our MBC cases were of rare histological type and uncommon presentation, namely synchronous bilateral invasive cribriform carcinoma and adenomyoepithelioma of the male breast.
\end{abstract}

Keywords: male breast cancer, invasive cribriform carcinoma of male breast, morbidity

\section{Introduction}

Male breast cancer (MBC) is an uncommon condition, accounting for $0.5-1 \%$ of all breast cancers [1]. Although MBC has its specific characteristics, the lack of large randomized trials of this disease resulted in the need of extrapolating data from female breast cancer to offer these patients an adequate medical management. Mean age at diagnosis of $\mathrm{MBC}$ is 67 years, men being diagnosed at an older age than women with this disease. Patients with MBC have lower survival rates (5-year-survival - 67\%) than postmenopausal women and relatively similar outcomes to premenopausal female patients

Received: December 2016; Accepted after review: March 2017; Published: March 2017.

${ }^{*}$ Corresponding author: Andrian Panuta, MD, PhD fellow, Ist Surgical Oncology Unit, lasi, Regional Cancer Institute, Romania, No 2-4 General Henry Mathias Berthelot Street, lasi, Romania.

E-mail: andrian.s.panuta@gmail.com with breast cancer [2]. There are certain genetic conditions that determine a higher lifetime risk of developing $\mathrm{MBC}$. The risk of $M B C$ is up to 50 times higher in individuals with Klinefelter's syndrome compared to individuals with normal karyotype. This chromosomal disorder is found in $7.5 \%$ of men with breast cancer. The lifetime risk of breast cancer is even higher (up to 100 times) in men with BRCA2 mutations [3]. Hyperestrogenism (due to hepatic insufficiency, testicular failure), hyperprolactinemia, alcohol intake and tobacco smoking, occupational risks (especially working in hot environments), slightly increase the risk of developing $\mathrm{MBC}$ [4]. Gynecomastia, a very common disorder in young men, has no influence on the risk of MBC [5]. The majority of malignant breast tumors in men are epithelial (carcinomas) with a ductal invasive (NST) histologic type. As the male mammary gland lacks lobular tissue, invasive lobular carcinoma of male breast is extremely rare. Most $\mathrm{MBC}$ are luminal $\mathrm{A}$ and $\mathrm{B}$ molecular subtypes. HER2neu is usually 
negative in MBC (in 95\% of cases) [6]. The objective of the current study is to present the clinical, biological and pathological features of male patients with breast cancer treated by a single surgical team.

\section{Patients and Methods}

We present the results of an observational retrospective study on the clinico-pathological features of patients with MBC which underwent surgical treatment in our unit - First Surgical Oncology Unit, lasi Regional Cancer Institute, Romania. From May 2012 to October 2016 we treated 10 male patients with breast cancer $(1.21 \%$ of all breast cancers in our casuistry - 822 cases).

\section{Clinico-pathological characteristics of the patient}

The median age of the patients was 61 years (range 30 to 81 years). Eight patients resided in urban and 2 in rural areas. To determine the possible risk factors for MBC we evaluated the comorbidities in our patients and found the following: metabolic disorders obesity in 3 cases, type II diabetes mellitus in 4 cases, cardiovascular diseases (arterial hypertension, arrhythmia, chronic cardiac insufficiency) in 4 cases, liver diseases in 3 cases (viral/toxic hepatitis, fatty change), respiratory diseases (chronic obstructive pulmonary disease, pulmonary tuberculosis sequels) in 3 cases, psychiatric diseases (schizophrenia, dementia and anxiety disorder) in 3 cases. No occupational exposure to heat, toxic chemicals or radiation was found. Of the $10 \mathrm{MBC}$ patients 3 were cigarette smokers, 4 moderate drinkers, and 1 heavy drinker. No suggestive family history or genetic predisposition was found in our MBC patients.

\section{Diagnosis}

All cases were diagnosed by physical examination, imaging (breast ultrasound and/or mammography) and preoperative tumor biopsy (core biopsy - a method of choice that providing data both on histological type and molecular (IHC) subtype of the breast lump used in 5 cases), fine needle aspiration (1 case) or intraoperative - extemporaneous pathologic exam (4 cases). Half of the patients were diagnosed in advanced stages and underwent neoadjuvant (preoperative) chemotherapy.

\section{Treatment}

Neoadjuvant chemotherapy was indicated by the multidisciplinary oncology committee in 5 MBC cases: in 3 cases $3-6$ cycles of doxorubicin and cyclophosphamide, in one case 5 cycles of FEC (epirubicin, cyclophosphamide and 5 -fluorouracil) and in one case 8 cycles of docetaxel monotherapy. Surgery was performed by a single surgical team, mainly consisting of Madden modified radical mastectomy ( 7 cases, of which in one case bilateral), simple mastectomy (2 cases in one patient with medical contraindications for radical surgery and one patient in which extemporaneous pathologic examination suggested an intracystic papillary carcinoma but the definitive pathological diagnosis was of adenomyoepithelioma of the breast), subcutaneous (skin-sparing) mastectomy (in one case with cribriform carcinoma in situ). No major postoperative complications were noticed. Length of hospital stay ranged between 5 and 13 days with a median of 8 days. All surgical specimens were submitted for complete pathological examination, immunohistochemical methods included. No specimen showed positive surgical margins (respecting the R0 resection oncological criterion). All cases were managed by a single oncological multidisciplinary committee (consisting of surgeons, radiotherapists, medical oncologists and pathologists). Cases with locally advanced breast tumors and positive axillary lymph nodes received adjuvant (postoperative) radiotherapy. All patients with hormone positive tumor underwent long term antiestrogen treatment (mainly Tamoxifen), and some of them received adjuvant chemotherapy. 


\section{Pathology}

Cribriform intraductal (in situ) carcinoma histology was revealed in one case. Eight of 10 patients showed invasive carcinomas (5 cases of ductal invasive (NST) carcinoma, two cases of mixed type invasive carcinoma (one case of invasive cribriform, tubular and NST carcinoma, and one case of micropapillary and NST carcinoma), one very rare case of bilateral synchronous invasive cribriform carcinoma (Figure 1), and one case of extremely rare histologic type adenomyoepithelioma of the male breast (with malignant behavior) (Table 1).

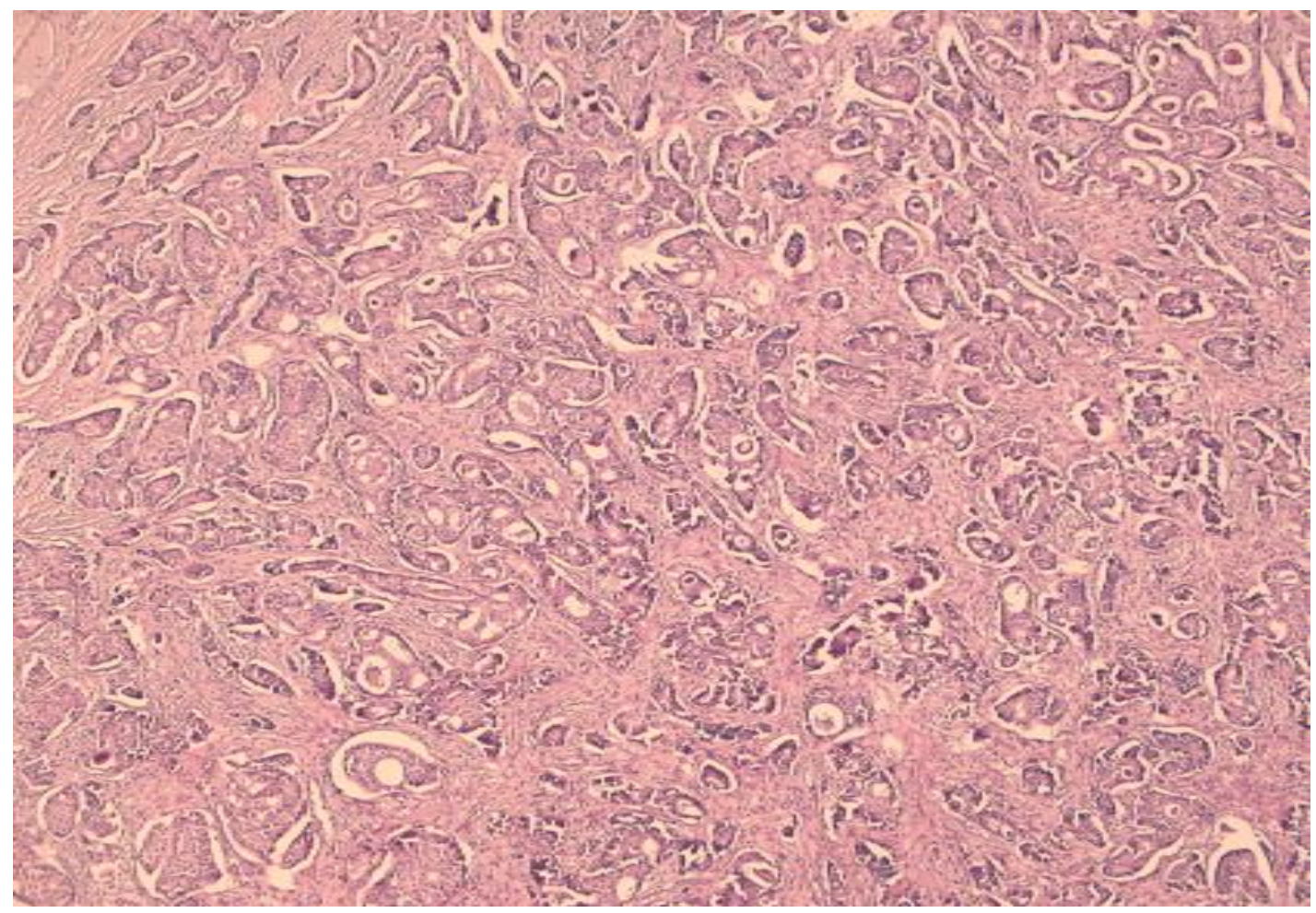

Fig. 1. Cribriform invasive breast cancer in male (HE, x100)

The breast tumors were diagnosed in the following stages: Tis $-10 \%, \mathrm{pT} 1-20 \%$, pT2 $30 \%$, pT3 - 20\%, pT4 - 20\%. Lymph node involvement was noted in $60 \%$ of cases. No metastatic disease was found at the time of surgery (Table 2).

Table 1. Histological types of MBC in our casuistry

\begin{tabular}{cc}
\hline Histologic type & MBC cases (number \%) \\
\hline Invasive Ductal carcinoma (NST) & $5(50)$ \\
\hline Invasive cribriform carcinoma & $1(10)$ \\
Mixed type invasive carcinoma & $2(20)$ \\
Cribriform intraductal carcinoma & $1(10)$ \\
Adenomyoepithelioma of the breast & $1(10)$ \\
\hline
\end{tabular}


Table 2. TNM stages in the studied MBC group

\begin{tabular}{|c|c|}
\hline Feature & MBC cases (number \%) \\
\hline Tumor size (T) & $\mathrm{n}(\%)$ \\
\hline pTis & $1(10)$ \\
\hline pT1 & $2(20)$ \\
\hline pT2 & $3(30)$ \\
\hline pT3 & $2(20)$ \\
\hline pT4 & $2(20)$ \\
\hline \multicolumn{2}{|l|}{ Nodal status (N) } \\
\hline $\mathrm{Nx}$ & $1(10)$ \\
\hline No & $3(30)$ \\
\hline $\mathrm{N} 1$ & $5(50)$ \\
\hline N2 & $1(10)$ \\
\hline \multicolumn{2}{|l|}{ Metastases (M) } \\
\hline$M x$ & $0(0)$ \\
\hline MO & $10(100)$ \\
\hline M1 & $0(0)$ \\
\hline \multicolumn{2}{|l|}{ Nottingham grade } \\
\hline Gx & $5(50)$ \\
\hline G1 & $2(20)$ \\
\hline G2 & $2(20)$ \\
\hline G3 & $0(0)$ \\
\hline Unknown & $1(10)$ \\
\hline \multicolumn{2}{|l|}{ Stages } \\
\hline 0 & $1(10)$ \\
\hline 1 & $0(0)$ \\
\hline$\| A$ & $3(30)$ \\
\hline IIB & $3(30)$ \\
\hline IIIA & $1(10)$ \\
\hline IIIB & $2(20)$ \\
\hline
\end{tabular}

Nottingham differentiation grade in 5 patients who underwent neoadjuvant chemotherapy was GX. In the remaining 5 cases that underwent upfront surgery the histologic grade was G2 (moderately differentiated) in 2 patients, G1 (well differentiated) in 2 patients, and unknown in one patient. Lymphovascular invasion was identified in one case and perineural invasion in three cases (Table 3).

Table 3. Lymphovascular Invasion and Perineural Invasion in the MBC cases

\begin{tabular}{cc}
\hline Feature & MBC cases (number \%) \\
\hline Lymphovascular Invasion & \\
Negative & $9(90)$ \\
Positive & $1(10)$ \\
Perineural Invasion & \\
Negative & $7(70)$ \\
Positive & $3(30)$ \\
\hline
\end{tabular}

Immunohistochemical examination revealed that in the majority of cases (nine cases) tumor cells had positive hormone receptors (Figure 2), negative (eight cases) or equivocal (two cases) HER2 expression, and high Ki67 (Figure 3) - proliferation index (ranging from $20 \%$ to $80 \%$ with a median of $38 \%)$. In our study group the most common molecular subtypes were luminal B (in seven cases), luminal A (two cases) and triple negative (one case) (Table 4). 
Table 4. Immunohistochemical features of MBC in our casuistry

\begin{tabular}{cc}
\hline Feature & MBC cases (number \%) \\
\hline Estrogen receptor & \\
\hline Positive & $9(90)$ \\
Negative & $1(10)$ \\
\hline Progesterone receptor & \\
\hline Positive & $8(80)$ \\
\hline Negative & $2(20)$ \\
\hline HER2 & \\
\hline Positive & $0(0)$ \\
\hline Negative & $8(80)$ \\
\hline Equivocal (2+) & $2(20)$ \\
\hline Molecular subtypes & \\
\hline Luminal A & $2(20)$ \\
\hline Luminal B & $7(70)$ \\
\hline Triple negative & $1(10)$ \\
\hline
\end{tabular}

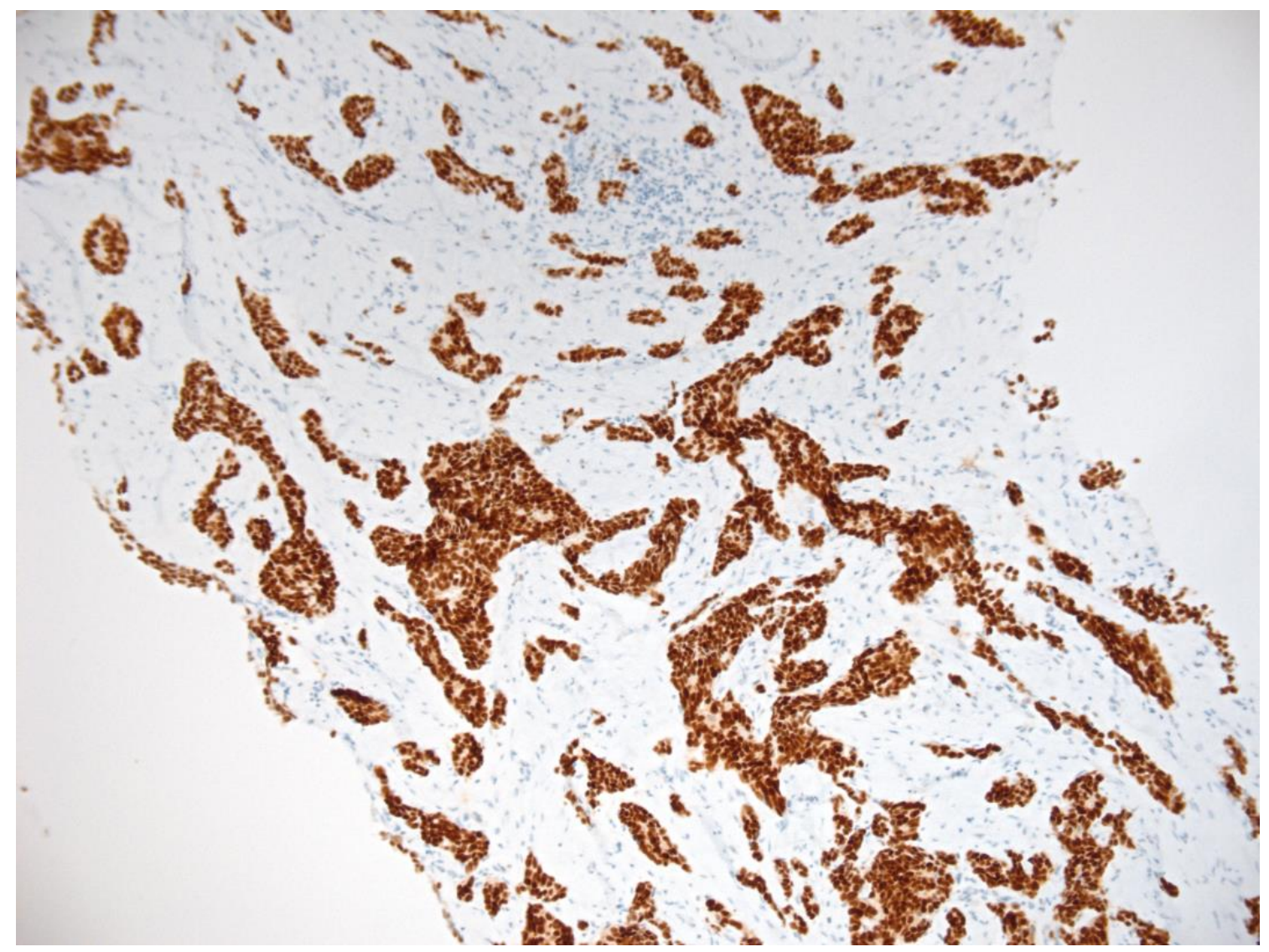

Fig. 2. Estrogen receptor positive in $90 \%$ of tumor cells in male breast cancer (IHC, Ab anti-ER, $x 100$ ) 


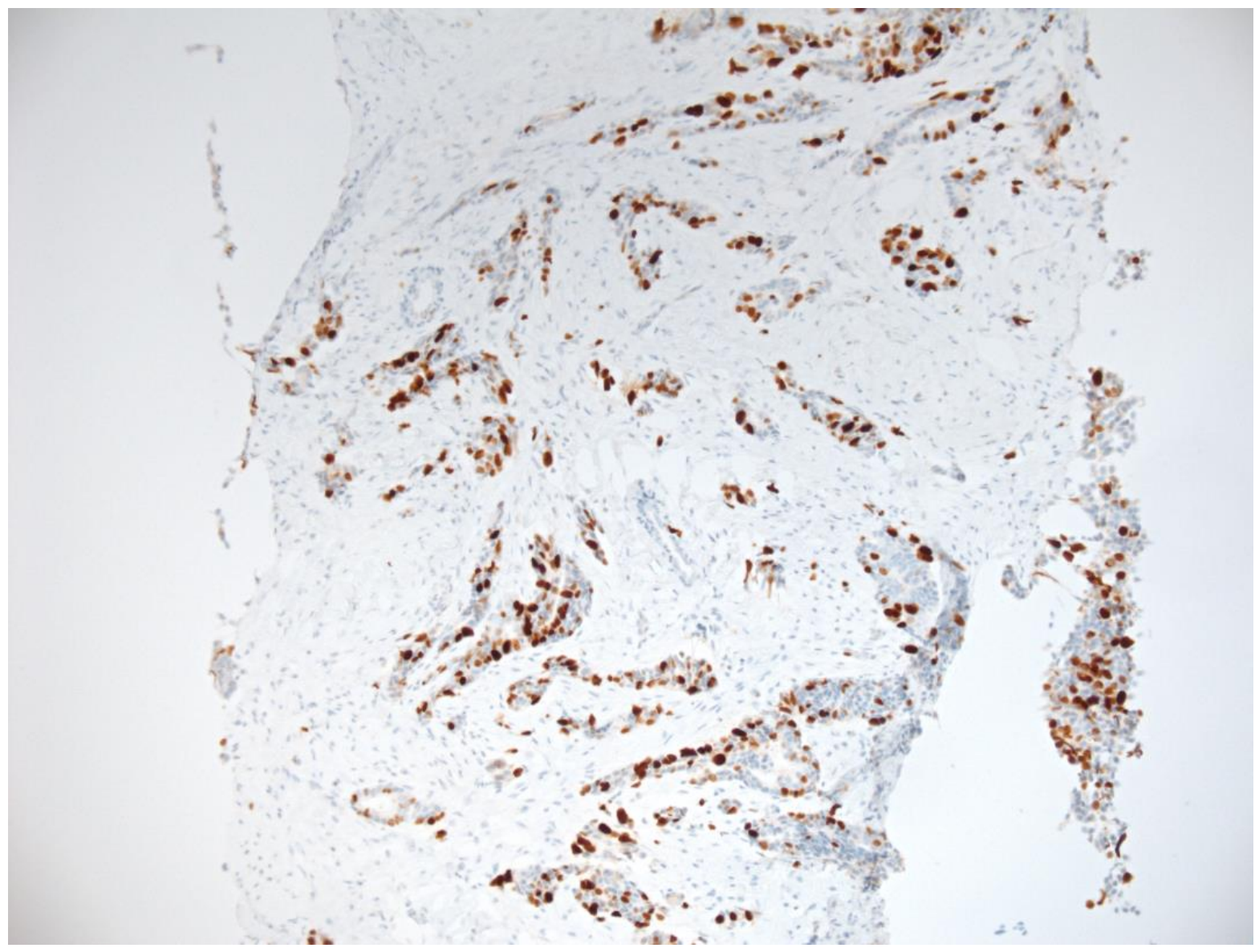

Fig. 3. Ki67 positive in 40-50\% of tumor cells in male breast cancer (IHC, Ab anti-Ki67, x100)

\section{Follow-up}

Our patients with MBC were followed-up every 3 months in first 2 years and every 6 months during the third-fifth years after surgery, by physical examination (both by a surgeon and an oncologist), tumor marker CA15.3 determination and bilateral breast and abdominal ultrasound. Also in some cases mammography, thoracic-abdominal CT and bone scintigraphy were performed in order to exclude/confirm the presence of local recurrence or distant metastases.

\section{Outcomes}

One patient received a simple mastectomy because of medical contraindications for radical surgery. He was afterwards readmitted to our unit for axillary lymphadenopathies and an axillary lymph node dissection, under local anesthesia, was performed. One patient died from metastases to the pleural cavity. The other 8 patients showed no signs of local or distant relapses.

\section{Discussions}

Breast cancer accounts for $0.1 \%$ of all male cancers. Although MBC shares some features with female breast cancer, it has different course patterns and its predictive factors are poorly understood [7]. Main prognostic factors in MBC are tumor size $(85 \%$ survival rate when tumor size is less than 2 $\mathrm{cm})$ and lymph node status $(50 \%$ higher risk of death in the patients with axillary node involvement) [8]. Histologic types of male breast cancer are mainly similar with those found in female patients. The most common type for MBC is invasive NST carcinoma (invasive carcinoma of no special type, previously known as invasive ductal carcinoma). Lobular carcinoma of the breast is very rare in male patients due to the absence 
of lobular tissue in male breast. Invasive cribriform carcinoma (ICC) of the male breast is a rare finding $(0.3 \%$ to $3.5 \%$ of all invasive breast carcinomas) $[9,10]$. The incidence of bilateral MBC is of about $2 \%$, and synchronous tumors are exceedingly rare [11]. Our cases of bilateral synchronous invasive cribriform carcinoma and adenomyoepithelioma of the male breast are extremely rare findings. In early stages of MBC surgery is the mainstay of treatment being curative and sufficient in some cases. Most MBCs are hormone receptor-positive (luminal $A$ and luminal B molecular subtypes). Long-term antiestrogen therapy (tamoxifen or aromatase inhibitors) significantly increases overall survival, prevents local recurrence and reduces the risk of developing metastatic disease in MBC. In locally advanced cases of MBC neoadjuvant chemotherapy is necessary in order to obtain operability. Adjuvant radiotherapy are indicated in MBC with large tumors, cutaneous involvement, invasion of the areola or subjacent muscles, axillary lymph-node metastases, positive margins on surgical specimen, multifocal disease, high grade tumors, high tumor proliferation rate, lymphovascular and perineural invasion and in those with negative-hormone-receptor disease. HER-2 amplification in MBC is rare $(2-15 \%)$. The effectiveness of targeted treatment with trastuzumab in MBC is uncertain due to the lack of controlled prospective studies, and may be used in patients with HER-2 positive breast cancers [3].

\section{References}

1. Groheux D, Hindié E, Marty M, et al. ${ }^{18}$ F-FDG$\mathrm{PET} / \mathrm{CT}$ in staging, restaging, and treatment response assessment of male breast cancer. Eur J Radiol 2014; 83(10):1925-1933.

2. Yu XF, Yang HJ, Yu Y, Zou DH, Miao LL. A prognostic analysis of male breast cancer (MBC) compared with post-menopausal female breast cancer (FBC). PLoS One 2015; 10(8):e0136670.

3. Sousa B, Moser E, Cardoso F. An update on male breast cancer and future directions for research and treatment. Eur J Pharmacol 2013; 717(1-3):71-83.

\section{Conclusions}

The lack of large multicentric randomized trials on MBC, due to its very low incidence, makes this disease poorly understood. This fact leads to the imperfection of existing management protocols of MBC. In our study the incidence of $\mathrm{MBC}$ was $1.32 \%$ from all cases of breast cancer (756 cases). The results of our retrospective study are similar to the literature reports except for the 2 cases of MBC with rare histological types and uncommon clinical presentations. In early stages MBC is curable and in some cases surgery alone is sufficient. A correctly performed multimodal treatment and follow-up by a specialized multidisciplinary team is necessary to increase the survival rates in men with breast cancer. Cases of MBC with rare histologic types represent a challenge for both the surgeon and the oncologist. Raising the breast cancer awareness among male population and medical personnel should lead to $M B C$ earlier diagnosis in order to improve its severe prognosis.

\section{Consent}

Written informed consent was obtained from the patients for publication of this study.

\section{Conflict of interest}

The authors declare that they have no competing interests. 
BIG/NABCG international male BC program. Cancer Res 2015; 75:S6-05.

7. Johansson I, Killander F, Linderholm B, Hedenfalk I. Molecular profiling of male breast cancer - lost in translation? Int J Biochem Cell Biol 2014; 53:526-535. Kahla PB, Cassaro S, Vladimir FG, Wayne MG, Cammarata A. Bilateral synchronous breast cancer in a male. Mt Sinai J Med 2005; 72(2):120-123.

8. Nishimura R, Ohsumi $\mathrm{S}$, Teramoto $\mathrm{N}$, Yamakawa T, Saeki T, Takashima S. Invasive cribriform carcinoma with extensive microcalcifications in the male breast. Breast Cancer 2005; 12(2):145-148.

9. Acevedo C, Amaya C, López-Guerra JL. Rare breast tumors: review of the literature. Rep Pract Oncol Radiother 2013; 19(4):267-274.

10. Farrokh F, Ansaripour E, Rastegar YF. Simultaneous bilateral male breast cancer: A case report and review of the literature. Iranian J Cancer Prev 2010; 3(4):199-203. 